\title{
Association of blood lactate with carotid atherosclerosis: The Atherosclerosis Risk in Communities (ARIC) Carotid MRI Study
}

\author{
Ghanshyam Palamaner Subash Shantha, MD, MPH ${ }^{1}$, Bruce Wasserman, MD2, Brad C. \\ Astor, MPH, PhD ${ }^{1,3}$, Josef Coresh, MD, PhD ${ }^{1,3}$, Fredrick Brancati, MD, MHS ${ }^{1,3}$, A. Richey \\ Sharrett, MD, DrPH ${ }^{1}$, and J. Hunter Young, MD, MHS ${ }^{1,3}$
}

Ghanshyam Palamaner Subash Shantha: gpalaman@jhsph.edu; Bruce Wasserman: bwasser@jhmi.edu; Brad C. Astor: bastor@jhsph.edu; Josef Coresh: coresh@jhu.edu; Fredrick Brancati: fbrancat@jhmi.edu; A. Richey Sharrett: rsharret@jhsph.edu; J. Hunter Young: jhyoung@jhmi.edu

${ }^{1}$ Department of Epidemiology, The Johns Hopkins Bloomberg School of Public Health, Baltimore, Maryland, USA

${ }^{2}$ The Russell H. Morgan Department of Radiology and Radiological Sciences, The Johns Hopkins University School of Medicine, Baltimore, Maryland, USA

${ }^{3}$ Department of Medicine, The Johns Hopkins University School of Medicine, Baltimore, Maryland, USA

\section{Abstract \\ Objectives-Cardiovascular risk factors such as aging, smoking, and insulin resistance may lead to atherosclerosis through various mechanisms of which their association with mitochondrial dysfunction may be one of them. In order to examine this hypothesis, we assessed the association between elevated blood lactate, a marker of mitochondrial dysfunction, and carotid atherosclerosis.}

Methods-From a total of 2066 participants from the Atherosclerosis Risk In Communities Carotid MRI study, 1496 were included for this analysis. Wall Thickness and Lipid core presence were measured using gadolinium-enhanced MRI. Blood lactate was categorized into quartiles (Q1: $<5.9 \mathrm{mg} / \mathrm{dl}$, Q2: 5.9 to $7.2 \mathrm{mg} / \mathrm{dl}$, Q3: 7.3 to $9.2 \mathrm{mg} / \mathrm{dl}$, and Q4: $>9.2 \mathrm{mg} / \mathrm{dl}$ ).

Results-Of the 1496 study participants, 763 (51\%) were females, 296 (19.8\%) African American, 539 (36\%) obese and $308(20.6 \%)$ had diabetes. There was a strong and graded association between lactate and wall thickness [Q1: $1.08 \mathrm{~mm}(95 \% \mathrm{CI}: 1.01 \mathrm{~mm}-1.15 \mathrm{~mm}), \mathrm{Q} 2$ : $1.33 \mathrm{~mm}$ (95\% CI: $1.19 \mathrm{~mm}-1.47 \mathrm{~mm}), \mathrm{Q} 3: 1.44$ (95\% CI: $1.34 \mathrm{~mm}-1.54 \mathrm{~mm})$ and Q4: 1.62 (95\% CI: $1.53 \mathrm{~mm}-1.71 \mathrm{~mm})$; p for trend $<0.001]$ after adjusting for age, gender, ethnicity, stature, body mass index (BMI), waist circumference, LDL, High sensitivity $\mathrm{C}$ reactive protein (HsCRP), statin use, thiazolodinedione use, hypertension, and diabetes. This association was attenuated, but still significant, after adjusting for a marker of insulin resistance, the triglyceride/ HDL ratio, [Q1: $0.96 \mathrm{~mm}(95 \% \mathrm{CI}: 0.82 \mathrm{~mm}-1.10 \mathrm{~mm})$, Q2: $1.17 \mathrm{~mm}$ (95\% CI: $1.08 \mathrm{~mm}-1.26$ $\mathrm{mm})$, Q3: $1.18 \mathrm{~mm}(95 \% \mathrm{CI}: 1.07 \mathrm{~mm}-1.29 \mathrm{~mm})$, Q4: $1.22 \mathrm{~mm}(95 \% \mathrm{CI}: 1.13 \mathrm{~mm}-1.31 \mathrm{~mm})$,

(C) 2013 Elsevier Ireland Ltd. All rights reserved.

Name and complete address for correspondence (address for reprints): Dr. J. Hunter Young MD, MHS, Department of Medicine and Department of Epidemiology, Johns Hopkins University, 2024E. Monument Street, Suite 2-625, Baltimore, MD 21287, USA. jhyoung@jhmi.edu, Phone: 410-502-5808, Fax: (410) 614-0588.

Publisher's Disclaimer: This is a PDF file of an unedited manuscript that has been accepted for publication. As a service to our customers we are providing this early version of the manuscript. The manuscript will undergo copyediting, typesetting, and review of the resulting proof before it is published in its final citable form. Please note that during the production process errors may be discovered which could affect the content, and all legal disclaimers that apply to the journal pertain.

Disclosures: None 
$\mathrm{p}$ for linear trend 0.039]. There was no association of lactate with lipid core presence after adjustment for wall thickness.

Conclusions-Blood lactate is associated with carotid atherosclerosis. Attenuation of the association with adjustment for triglyceride/HDL ratio, a marker of insulin resistance, suggests that lactate's association with carotid atherosclerosis may be related to insulin resistance.

\section{Keywords}

atherosclerosis; carotid arteries; plaque; epidemiology; lactate

Recent evidence implicates mitochondrial dysfunction in the pathogenesis of atherosclerosis. Mitochondrial dysfunction may be related to atherosclerosis due its local effect on the production of reactive oxygen species (ROS) [1] and subsequent oxidized LDL, endothelial cell dysfunction, and increased vascular cell proliferation, a milieu suitable for atherogenesis [1]. In addition, mitochondrial dysfunction is associated with cardiovascular risk factors such as insulin resistance [2, 3, 4]. Furthermore, mitochondrial dysfunction is considered an intermediary through which common cardiovascular risk factors such as aging, hyperglycemia, hyperhomocystenemia and smoking may lead to atherosclerosis [5].

High levels of lactate during exercise indicate low aerobic capacity [6]. In resting individuals, elevated blood lactate is used to indicate primary mitochondrial dysfunction (e.g. hereditary enzyme defects) [2] and insufficient oxygen delivery (e.g. hypoxia and ischemia) [7]. Elevated levels of blood lactate are also indicative of more subtle degrees of low oxidative capacity that occur with obesity and insulin resistance $[8,9,10,11]$. Given the accumulating evidence linking mitochondrial dysfunction to atherosclerosis, we hypothesized that mitochondrial dysfunction, as assessed by higher levels of blood lactate, is associated with sub-clinical, stable, chronic atherosclerotic lesions in the carotid vasculature among the participants of Atherosclerosis Risk in Communities (ARIC) carotid Magnetic Resonance Imaging (MRI) Study.

\section{Methods and Results}

\section{Study Population}

Study participants were selected from the Atherosclerosis Risk in Communities (ARIC) study cohort [12]. The ARIC study was approved by the institutional review board of the John's Hopkins University, Baltimore, MD. The ARIC study is a prospective predominantly biracial observational cohort of 15,792 individuals aged 45 to 64 years recruited from a probability sample of four communities (Forsyth County, NC; Jackson, Miss; suburban Minneapolis, Minn; and Washington County, MD). Participants took part in clinic examinations, starting with a baseline visit between 1987 and 1989 and continuing with three follow-up examinations at approximately 3-year intervals, at which point carotid artery intimal medial thickness (IMT) was measured with B-mode ultrasound [13]. Participants in the ARIC carotid magnetic resonance (MR) imaging study were selected from among the surviving ARIC study participants with a disproportionate stratified sampling plan on the basis of the most recent IMT and field center. The goal was to recruit 1200 participants with high values of maximum carotid artery IMT \{maximum over six sites: left and right common carotid artery (CCA), carotid bifurcation, and internal carotid artery (ICA) $\}$ at their most recent ultrasound examination (examination 3 or 4, performed between 1993 and 1995 or between 1996 and 1998, respectively) and 800 individuals who were selected from among the remaining eligible participants. Field center specific cutoff points of carotid IMT were adjusted over the recruitment period to approximately achieve this goal, with $100 \%$ 
sampling above the cutoff point and a sampling fraction $(16.5 \%-25.5 \%)$ below the cutoff point. The cutoff point was $1.135 \mathrm{~mm}$ in Forsyth County, NC; $1.000 \mathrm{~mm}$ in Jackson, Miss; $1.280 \mathrm{~mm}$ in suburban Minneapolis, Minn; and $1.215 \mathrm{~mm}$ in Washington County, MD; representing the 73rd, 69th, 73rd, and 68th percentiles of maximal IMT from examination 4, respectively. Persons who were not black or white ( $\mathrm{n}=10$ in Forsyth County, NC) were excluded from the selection process, as were those without IMT measurements at examination 3 or $4(n=1428)$. In addition, participants with contraindications to MR imaging or contrast media were excluded $(n=206)$, as were participants who had difficulty understanding questions or providing informed consent $(n=51)$, those who had undergone prior carotid revascularization on either side (for the low IMT group) or on the side selected for imaging (for the high IMT group) $(\mathrm{n}=58)$, and those who could not participate because of a self-reported health problem $(n=486)$ or another reason $(n=36)$. A total of 4306 persons were contacted and invited to participate in the study. Of these, 1403 declined, 837 were ineligible per above criteria, and 2066 participated (1250 with high IMT, 816 with low IMT). Of the 2066 participants, 1939 had undergone MR imaging, and 1769 MR studies were of sufficient quality and adherence to the MR imaging protocol to merit inclusion. Of these 1639 participants who had no missing data for all MRI variables of interest and lactate measures (described later) were finally included.

\section{MR Imaging Methods}

A standard MRI protocol was used for all participants and performed on 1.5T scanners (Excite platform, GE Medical Systems, Forsyth County, Jackson, and Washington County, USA; Symphony Maestro, Siemens Medical Solutions, Minneapolis, USA) using bilateral four-element phased array carotid coils (Machnet, The Netherlands). Fourteen MRI technologists, trained centrally and certified by the MRI Reading Center, acquired the scans. Total protocol time was less than one hour $[14,15]$. Black-blood MR images were acquired by using a two-dimensional cardiac-gated double inversion-recovery fast spin-echo sequence that was based on a standardized protocol [14] and the following parameters: field of view, $13 \mathrm{~cm}$; section thickness, $2 \mathrm{~mm}$; matrix, $256 \times 224$; echo train length, 10 ; one signal acquired; and acquired resolution, $0.51 \times 0.58 \times 2 \mathrm{~mm}$. Three long-axis black-blood MR imaging sections (repetition time msec/echo time $\mathrm{msec} /$ inversion time msec, two R-R intervals/5/600) were acquired through each carotid artery bifurcation by using a time-offlight MR angiogram as a scout image (Figure 1). The black-blood MR image that best depicted the bifurcation, including the flow divider, was used to orient all transverse T1weighted black-blood MR images. The transverse sections were acquired (repetition time msec/echo time msec, one R-R interval/5) with chemical suppression of fat signal before and 5 minutes after the intravenous injection of gadodiamide $(0.1 \mathrm{mmol}$ per kilogram of body weight; Omniscan, GE Healthcare, Prineton, NJ) with a power injector. The inversion time was changed to $200 \mathrm{msec}$ for postcontrast images to enable blood signal suppression. A transverse T1-weighted black-blood MR section was acquired through each distal common carotid artery, positioned $1.5 \mathrm{~cm}$ below the flow divider, and oriented perpendicular to the vessel axis (Figure 1). Sixteen contrast enhanced transverse T1-weighted black-blood MR images (total longitudinal coverage, $3.2 \mathrm{~cm}$ ) were then acquired through the carotid bifurcation found to have the greatest maximum wall thickness at the participant's most recent US examination. If the contralateral carotid bifurcation wall appeared thicker to the MR imaging technologist on the MR angiographic source images or more stenotic on the MR angiographic maximum intensity projection images, however, this vessel was selected for the multi-section acquisition. The sections were oriented perpendicular to the vessel axis and centered at the thickest part of the carotid bifurcation or through the flow divider if no plaque was present. Analyses were repeated after excluding 34 vessels that were oriented obliquely ( $25 \%$ ), with nearly identical results. We used internal carotid artery (ICA) 
measurements from the section located one section $(2 \mathrm{~mm})$ above the flow divider for the present analysis.

\section{Image Analysis}

Seven analysts were trained to interpret the MR images with semi-automated software (Vessel MASS; Division of Image Processing, Radiology Department, Leiden University Medical Center, Leiden, the Netherlands). The analysts were blinded to the characteristics of the study participants. All studies were graded for image quality and adherence to the imaging protocol, and studies that failed were not analyzed. Post-contrast black-blood MR images were analyzed by using semi-automated analysis software, as described previously $[14,15]$. Over the course of the study, 61 randomly selected participants repeated the entire clinic visit including the MRI exam within four to eight weeks to estimate total MRI measurement error from both scan acquisition and reader variability. Both the MRI technologists and the readers were blinded to the repeat participants. Reader reliability was estimated by randomly reassigning scans for interpretation by the same $(\mathrm{N}=53)$ or different $(\mathrm{N}=111)$ reader. The target interval between readings by the same analyst was at least 90 days to minimize the influence of recall. For linear measurements, our reliability estimates were 0.83 for Wall Thickness (WT), 0.90 for Lumen Area (LA), and 0.89 for identifying lipid core presence [15].

\section{Description of MRI variables used for this analysis}

From all the MRI variables measured, we selected 3 variables which were found to have high reliability between observers and between repeat scans as reported in a previous study [15]. They are: 1) Wall Thickness (WT) (mm): denotes maximum internal carotid artery wall thickness of 12 segments of the slice $2 \mathrm{~mm}$ above the flow divider, 2) Lumen Area (LA) - lumen area of the left common carotid artery slice. Since, lumen area measured on both left and the right common carotid arteries had similar measures, left common carotid artery lumen area has been used arbitrarily, 3) Lipid core presence (LP) (\%): presence/ absence of core at any of the eight slices. This is a categorical variable. Due to resolution constraints of the MRI scan lipid core assessment was restricted to those 1131 participants whose maximum wall thickness was $>1.5 \mathrm{~mm}$. Only 4 lipid cores were excluded using this cut point. Among these 1131 participants included in this analysis 542 participants had lipid rich cores. Variables such as total wall volume, maximum wall thickness, vessel wall area, mean minimum fibrous cap thickness etc. which were used for analysis in previous studies [16] were not included in our analysis because the study by Wasserman et al. [15] had shown that these variables had poor inter-observer and inter-scan reliability.

\section{Lactate and other lab variables collection}

The measurement of lactate was approved by the ARIC Steering Committee as an ARIC ancillary study. Since Metformin increases blood lactate levels, an additional 143 subjects with type 2 diabetes (26\%) were excluded if they were taking Metformin during the 4 weeks prior to the carotid MR imaging [17]. Hence, from the 1639 included the sample size went down to 1496 participants after exclusion of these 143 subjects. The core examination procedures are identical to those previously established by ARIC [12]. Briefly, a 90-min examination was performed in the morning after a 12-h fast. After informed consent, trained technicians performed anthropometry measurements and obtained urine and blood samples. Blood samples were collected in 10-ml potassium-EDTA (lavender top) tubes (Becton, Dickinson and Company). Following each blood draw, blood was iced immediately and kept between 0 and $8^{\circ} \mathrm{C}$ prior to centrifugation $\left(3000 \mathrm{~g}\right.$ for $20 \mathrm{~min}$ at $4^{\circ} \mathrm{C}$ ). Within $30 \mathrm{~min}$ of the blood draw, plasma was aliquoted and stored at $-70^{\circ} \mathrm{C}$ at the field centers. Frozen samples where then shipped by Federal Express Priority Overnight mail to the ARIC central laboratories for long-term storage and analysis. Plasma lactate was measured using an 
enzymatic reaction to convert lactate to pyruvate using a Roche Hitachi 911 auto-analyzer [18]. In order to assess the reliability of the lactate measurements, we performed within-visit and repeat-visit assessments of plasma lactate. The quality control analysis of blind replicate samples in 117 pairs demonstrated a within-visit coefficient of reliability of 0.93 with a coefficient of variation of $9.2 \%$. The quality-control analysis of samples drawn from 61 individuals on separate days demonstrated a day-to-day coefficient of repeatability of 0.55 with a coefficient of variation across the two visits of $24.7 \%$. The mean difference between the repeat and study measurement was small (mean absolute difference $=0.11 \mathrm{mg} / \mathrm{dl}$ ) and not significantly different from zero [95\% confidence interval (CI) $-0.82,0.60 \mathrm{mg} / \mathrm{dl}]$. Furthermore, no systematic bias was detected (test for the proportion of positive differences $=50 \%, \mathrm{P}=0.694)$.

\section{Other co-variates}

Other variables of interest included age, ethnicity, gender, ARIC field center, triglycerides, high-density lipoprotein (HDL) and low-density lipoprotein (LDL) cholesterol, Triglyceride/ HDL ratio, body mass index (BMI), waist circumference, and smoking status (current). Details have been previously described for measurement of plasma lipids [19], fasting glucose [20] and determination of BMI ( $\mathrm{kg} / \mathrm{m} 2)$ [21]. To ascertain medication use, participants were asked to bring containers of current medications to the visit. Antihypertensive medications, anti-diabetic medications and statin use was accordingly determined.

\section{Statistical Methods}

Data are expressed as means and $95 \%$ CIs or medians and interquartile range for continuous variables and as number (n) and percentage (\%) for categorical variables. All statistical analyses incorporated the disproportionate stratified sampling design, for estimation, testing and CIs, using Stata 11 [22]. Sampling weights were based on the probability of being selected from each field center based upon the high-IMT status of each participant. The sampling weight among high-IMT subjects was approximately equal to 1 for all field centers. The sampling weights for individuals in the remainder of the IMT distribution varied according to field center, ranging from 3.9 to 6.1. MRI cannot clearly characterize plaque characteristics when the IMT is low. Therefore, high IMT participants were oversampled from the ARIC population in order to maximize the proportion of participants with plague characteristics that can be measured given the resolution limits of MRI. In other words, high IMT participants were over-sampled to increase the yield of MRI findings with regards to carotid atherosclerosis. The advantage of the stratified sampling design is that it allowed for oversampling of high-IMT subjects, while enabling us to make generalizable inferences to the ARIC base population using sampling weights. The trade-off is a loss of precision of the estimates relative to analysis of the entire study population. Supplemental Figure 2 displays the relationship between log lactate and $\log$ wall thickness after adjustment with the sampling weights.

Characteristics of subjects participating in the ARIC carotid MRI study were first compared across lactate quartiles. To assess for linear trends across quartiles, accounting for the sampling distribution and non-linearity in the lactate distribution, survey-weighted logistic regression and survey-weighted linear regression were utilized, treating the variable of interest (patient characteristics) as the dependent variable and the median lactate value for each quartile as a continuous independent variable. Then similarly, survey weighted linear regression analysis was performed treating the MRI variables of interest namely WT and LA as dependent variables and the median lactate value for each quartile as a continuous independent variable. Model 1, was adjusted for demographic variables age, gender, ethnicity and field center. Model 2 included variables height, height ${ }^{2}$, BMI and waist 
circumference in addition to those variables included in model 1 . These associations were adjusted for height and height ${ }^{2}$ because carotid wall thickness and caliber of carotid lumen are known to increase with height [23]. Model 3 included model 2 variables and the variables smoking, hypertension diagnosis, diabetes diagnosis, HbA1C\%, LDL and HsCRP. Model 4 included model 3 variables and the variables statin use, thiazolidinedione use. Model 5 (fully adjusted model) included model 4 variables and Triglyceride/HDL ratio. Further, these analyses were performed stratified for gender, ethnicity, obesity, and diabetes. For these stratified analyses respective stratification variables were removed from the models. Similarly, survey-weighted logistic regression analysis was performed to assess the odds of having a lipid core (LP) (lactate quartile 1: $<5.9 \mathrm{mg} / \mathrm{dl}$ was considered the reference). Limited resolution of MRI imaging limited detection of smaller lipid cores, and as a consequence, cores were almost never detected in wall segments thinner than $1.5 \mathrm{~mm}$ (there were only 4 exceptions), and the frequency of detecting cores increased monotonically with increasing wall thickness. Since this is counter-intuitive (vessels evaluated pathologically are not absent cores when they are less than $1.5 \mathrm{~mm}$ thick, and cores do not increase in prevalence with thicker plaques), it is thought to be an artifact of inadequate resolution. Thus, to study factors associated with cores we utilize analyses adjusting for the maximum thickness of the wall. A P-value of $<0.05$ was considered statistically significant.

\section{Results}

The final study cohort consisted of 1496 participants. Mean age was 70.4 years, $51 \%$ were females and $19.8 \%$ were African Americans (Table 1). $19 \%$ were current smokers. Mean BMI was $28.8 \mathrm{~kg} / \mathrm{m}^{2}$ and mean waist circumference was $100.9 \mathrm{cms} .36 \%$ of the cohort was obese (BMI $\geq 30 \mathrm{~kg} / \mathrm{m} 2$ ). $20.6 \%$ had diabetes.

The median value for blood lactate was $7.2 \mathrm{mg} / \mathrm{dl}$ [IQR: $5.9-9 \mathrm{mg} / \mathrm{dl}]$ and $97 \%$ of our participants had lactate within the normal range $(4.5-19.8 \mathrm{mg} / \mathrm{dl})$. Age and gender did not vary across lactate quartiles. The proportion of African Americans and the proportion of participants with diabetes, hypertension, and obesity were greater in higher quartiles. Furthermore, BMI, waist circumference, glucose, triglycerides, LDL and the triglyceride/ HDL ratio were higher in higher lactate quartiles (Table 1).

The MRI variables included wall thickness, lumen area, and the presence of a lipid rich core. Lactate was not associated with lumen area. The association with wall thickness, however, was strong, graded, and independent of demographic, anthropometric, and CVD risk factors (Q1: $1.08 \mathrm{~mm}$ (0.034), Q2: $1.33 \mathrm{~mm}$ (0.071), Q3: 1.44 (0.054) and Q4: $1.62(0.044)$; p for trend $<0.001$; (Table 2)).

When stratified by gender and race, a similar strong, graded and independent association between lactate and wall thickness was observed [males (Q1: $1.04 \mathrm{~mm}(0.046), \mathrm{Q} 2: 1.35$ $\mathrm{mm}(0.031), \mathrm{Q} 3: 1.52(0.068)$ and Q4: $1.71(0.077)$; $\mathrm{p}$ for trend $<0.001$ ], females (Q1: 1.03 $\mathrm{mm}(0.028), \mathrm{Q} 2: 1.38 \mathrm{~mm}(0.053), \mathrm{Q} 3: 1.47$ (0.081) and Q4: $1.68(0.064) ; \mathrm{p}$ for trend $<0.001$ ], Whites (Q1: $1.08 \mathrm{~mm}(0.039), \mathrm{Q} 2: 1.33 \mathrm{~mm}(0.031), \mathrm{Q} 3: 1.44(0.068)$ and Q4: 1.62 (0.077); p for trend <0.001] and African Americans (Q1: $1.08 \mathrm{~mm}(0.041)$, Q2: $1.33 \mathrm{~mm}$ (0.073), Q3: 1.44 (0.081) and Q4: $1.62(0.055)$; $\mathrm{p}$ for trend <0.001] (supplemental tables 1 and 2). Also, this association was similar in the obese (Q1: $1.07 \mathrm{~mm}(0.040), \mathrm{Q} 2: 1.28 \mathrm{~mm}$ (0.033), Q3: $1.39(0.026)$ and Q4: $1.51(0.057)$; $\mathrm{p}$ for trend <0.001) and the diabetics (Q1: $1.01 \mathrm{~mm}(0.041), \mathrm{Q} 2: 1.16 \mathrm{~mm}(0.073), \mathrm{Q} 3: 1.31(0.081)$ and Q4: $1.38(0.055) ; \mathrm{p}$ for trend $<0.001$ (supplemental table 3). 
After adjusting for triglyceride/HDL ratio, the association between wall thickness and lactate was attenuated but still significant (Q1: $0.096 \mathrm{~mm}(0.071), \mathrm{Q} 2: 1.17 \mathrm{~mm}(0.046), \mathrm{Q} 3$ : 1.18 (0.055) and Q4: 1.22 (0.048); p for trend 0.039) (table 2). This was true among males, females, whites, African Americans, diabetics and the obese (supplemental tables 1,2 and 3)

Among the 1131 participants with a maximum wall thickness $>1.5 \mathrm{~mm}, 542$ participants had lipid rich cores. $15 \%$ were in Q1, $18 \%$ in Q2, 27\% in Q3 and 40\% were in Q4. However, the association between lactate and lipid core presence was not significant after adjustment for wall thickness.

\section{Discussion}

We have shown that blood lactate measured at rest has a strong and graded association with carotid wall thickness. This association is independent of traditional cardiovascular risk factors. As observed with most cardiovascular risk factors [24], lactate's association with lipid core is not independent of wall thickness.

Carotid atherosclerotic plaque burden and plaque composition are associated with clinical events including stroke [24, 25]. Wall thickness measured using MRI and carotid intima media thickness measured using ultrasonography are markers of carotid atherosclerotic plaque burden and are strongly associated with stroke risk [24, 25, 26]. Risk factors independently associated with wall thickness include age, male sex, white race, total and low-density lipoprotein cholesterol, diabetes, hypertension, and smoking [24]. In addition to plaque burden, the composition of the atherosclerotic plaque confers risk of clinical events $[26,27]$. Specifically, plaques that have a large necrotic lipid rich core and a thin fibrous cap are less stable and prone to rupture, leading to local thrombosis and downstream ischemia $[28,29]$.

Our results suggest that resting blood lactate may be higher in patients with established carotid atherosclerosis, independent of other cardiovascular risk factors. Clinically, elevated lactate is used to indicate energy imbalance due to insufficient oxygen delivery (e.g. hypoxia and ischemia) and due to primary mitochondrial dysfunction (e.g. hereditary enzyme defects). The extent and timing of an increase in blood lactate with exercise is a robust measure of aerobic capacity or fitness. In a population-based sample of resting individuals, lactate variation within the normal range may reflect variation in adiposity, insulin resistance and oxidative capacity [11]. Many lines of evidence suggest that low oxidative capacity is associated with atherosclerosis risk factors including insulin resistance. This evidence includes the association of insulin resistance and type 2 diabetes with maternally inherited forms of diabetes [2, 3], increased glycolysis in muscle [4, 30, 31] decreased mitochondrial size and density $[32,33]$, decreased oxidative gene expression $[34,35]$ decreased oxidative phosphorylation and decreased aerobic capacity [36, 37, 38]. Therefore, lactate's association with plaque burden may reflect the impact of risk factors associated with insulin resistance, as reflected by the attenuation of the association by the triglyceride/HDL ratio, a marker of insulin resistance. We had previously established a strong, independent and graded association between lactate and type 2 diabetes mellitus [11]. In this study, however, the association of lactate with plaque burden remained robust after adjusting for diabetes and glycemic control suggesting that lactate may indicate factors other than insulin resistance.

Mitochondrial dysfunction, as reflected by higher lactate levels, increases production of reactive oxygen species (ROS), resulting in increased oxidation of LDL and facilitating the formation of plaque. Furthermore, mitochondrial dysfunction leads to endothelial cell dysfunction and apoptosis and increased vascular smooth muscle proliferation, a milieu suitable for plaque accumulation. Therefore, mitochondrial dysfunction may be an 
intermediary though which many of the conventional cardiovascular risk factors such as age, smoking, hyperglycemia, and hyperhomocystinemia may operate to cause atherosclerosis. Therefore, higher blood lactate levels may reflect the impact of local mitochondrial dysfunction on plaque formation.

The association of blood lactate with plaque burden could also be due to reverse causation. Excessive accumulation of plaque results in arterial stenosis, compromise of blood flow, and increased lactate production. As shown by Astor and colleagues, however, the carotid arteries have good remodeling capacity in the setting of plaque accumulation [23]. The luminal area of the internal carotid artery does not start to decrease until the wall's thickness is at the highest level of its range. Furthermore, we show that lumen area is not associated with lactate level. These observations do not rule out the possibility that carotid wall thickness correlates with wall thickness in other less compliant arteries and, therefore, hypoperfusion of their tissue beds.

\title{
Limitations
}

To our knowledge, this study is the first to demonstrate an association between blood lactate and large vessel atherosclerosis. Our study has several important limitations. First, the crosssectional study design precludes examination of the temporal relationship between blood lactate and carotid atherosclerosis. Therefore, reverse causation cannot be excluded. Second, blood lactate can be artificially elevated due to ongoing glycolysis following blood draw. This effect was minimized through the rapid processing and cooling of blood samples. Third, the day-to-day repeatability of resting blood lactate is moderate, reflecting known diurnal variation in lactate levels and sensitivity of lactate to changes in the metabolic state. Despite this variation, lactate was strongly associated with carotid atherosclerosis. Fourth, of the 308 in the cohort with diabetes, 143 receiving metformin were excluded from the analysis. Due to this exclusion, the sample of diabetic participants in our analysis may be enriched with conditions, such as renal impairment, that impact the decision to treat with metformin. Finally, we recognize that lactate is an indirect indicator of oxidative capacity. Therefore, other factors could be responsible for lactate's association with atherosclerosis.

In conclusion, lactate is strongly associated with carotid atherosclerosis and the association is independent of traditional cardiovascular risk factors. A portion of the association may be due to the impact of insulin resistance and associated factors on atherosclerosis accumulation. In addition, mitochondrial dysfunction may have direct effects on plaque development and stability through increased production of oxidized LDL, proliferation of vascular smooth muscle, and apoptosis of endothelial cells. Additional work should further explore the relationship between markers of mitochondrial dysfunction, atherosclerosis, and subsequent clinical events.

\section{Supplementary Material}

Refer to Web version on PubMed Central for supplementary material.

\section{Acknowledgments}

\author{
None \\ Sources of funding: The Atherosclerosis Risk in Communities Study is carried out as a collaborative study \\ supported by National Heart, Lung, and Blood Institute contracts (HHSN268201100005C, HHSN268201100006C, \\ HHSN268201100007C, HHSN268201100008C, HHSN268201100009C, HHSN268201100010C, \\ HHSN268201100011C, and HHSN268201100012C). The authors thank the staff and participants of the ARIC \\ study for their important contributions. The first author is supported in part by a NIH/NHLBI T32HL007024
}


Cardiovascular Epidemiology Training Grant for his research training at the Johns Hopkins Bloomberg School of Public Health. JHY is supported in part by an NIH/NHLBI RO1DK085458 grant.

\section{Reference}

1. Madamanchi NR, Runge MS. Mitochondrial dysfunction in atherosclerosis. Circ Res. 2007; 100(4): 460-473. [PubMed: 17332437]

2. Robinson BH. Lactic acidemia and mitochondrial disease. Mol Genet Metab. 2006; 89(1-2):3-13. [PubMed: 16854608]

3. Gerbitz KD, Gempel K, Brdiczka D. Mitochondria and diabetes-genetic, biochemical, and clinical implications of the cellular energy circuit. Diabetes. 1996; 45:113-126. [PubMed: 8549853]

4. Simoneau JA, Colberg SR, Thaete FL, Kelley DE. Skeletal muscle glycolytic and oxidative enzyme capacities are determinants of insulin sensitivity and muscle composition in obese women. FASEB J. 1995; 9:273-278. [PubMed: 7781930]

5. Puddu P, Puddu GM, Cravero E, De Pascalis S, Muscari A. The emerging role of cardiovascular risk factor-induced mitochondrial dysfunction in atherogenesis. J Biomed Sci. 2009; 16:112. [PubMed: 20003216]

6. Tanaka H, Seals DR. Endurance exercise performance in Masters athletes: age-associated changes and underlying physiological mechanisms. The Journal of Physiology. 2008; 586(1):55-63. [PubMed: 17717011]

7. Jansen TC, van Bommel J, Bakker J. Blood lactate monitoring in critically ill patients: a systematic health technology assessment. Crit Care Med. 2009; 37(10):2827-2839. [PubMed: 19707124]

8. Avogaro A, Toffolo G, Miola M, Valerio A, Tiengo A, Cobelli C, et al. Intracellular lactate- and pyruvate-interconversion rates are increased in muscle tissue of non-insulin-dependent diabetic individuals. J. Clin. Invest. 1996; 98(1):108-115. [PubMed: 8690781]

9. Sandqvist MM, Eriksson JW, Jansson PA. Increased lactate release per fat cell in normoglycemic first-degree relatives of individuals with type 2 diabetes. Diabetes. 2001; 50(10):2344-2348. [PubMed: 11574418]

10. Hosogai N, Fukuhara A, Oshima K, Miyata Y, Tanaka S, Segawa K, et al. Adipose tissue hypoxia in obesity and its impact on adipocytokine dysregulation. Diabetes. 2007; 56:901-911. [PubMed: 17395738]

11. Crawford SO, Hoogeveen RC, Brancati FL, Astor BC, Ballantyne CM, Schmidt MI, et al. Association of blood lactate with type 2 diabetes: the Atherosclerosis Risk in Communities Carotid MRI Study. Int J Epidemiol. 2010; 39(6):1647-1655. [PubMed: 20797988]

12. The ARIC investigators. The Atherosclerosis Risk in Communities (ARIC) study: design and objectives - the ARIC investigators. Am J Epidemiol. 1989; 129(4):687-702. [PubMed: 2646917]

13. Heiss G, Sharrett AR, Barnes R, Chambless LE, Szklo M, Alzola C. Carotid atherosclerosis measured by B-mode ultrasound in populations: associations with cardiovascular risk factors in the ARIC study. Am J Epidemiol. 1991; 134(3):250-256. [PubMed: 1877584]

14. Wasserman BA, Smith WI, Trout HH 3rd, Cannon RO 3rd, Balaban RS, Arai AE. Carotid artery atherosclerosis: in vivo morphologic characterization with gadolinium-enhanced double-oblique MR imaging initial results. Radiology. 2002; 223(2):566-573. [PubMed: 11997569]

15. Wasserman BA, Astor BC, Sharrett AR, Swingen C, Catellier D. MRI measurements of carotid plaque in the atherosclerosis risk in communities (ARIC) study: methods, reliability and descriptive statistics. J Magn Reson Imaging. 2010; 31(2):406-415. [PubMed: 20099354]

16. Virani SS, Nambi V, Hoogeveen R, Wasserman BA, Coresh J, Gonzalez F 2nd, et al. Relationship between circulating levels of RANTES (regulated on activation, normal T-cell expressed, and secreted) and carotid plaque characteristics: the Atherosclerosis Risk in Communities (ARIC) Carotid MRI Study. Eur Heart J. 2011; 32(4):459-468. [PubMed: 20943669]

17. Davis TM, Jackson D, Davis WA, Bruce DG, Chubb P. The relationship between metformin therapy and the fasting plasma lactate in type 2 diabetes: The Fremantle Diabetes Study. Br J Clin Pharmacol. 2001; 52:137-144. [PubMed: 11488769]

18. Barhan D, Trinder P. An improved colour reagent for the determination of blood glucose by the oxidase system. Analyst 97. 1972; 142 
19. ARIC Coordinating Center. [Last accessed 15th June 2012] Atherosclerosis Risk in Communities Study Protocol: Lipid and Lipoprotein Determinations, Manual 8. ARIC study website. 1994. http://www.cscc.unc.edu/aric/pubuse/manual/Lipid_and_Lipoprotein_Determinations.1_8.pdf.

20. ARIC Coordinating Center. [Last accessed 15th June 2012] Atherosclerosis Risk in Communities Study Protocol: Clinical Chemistry Determinations, Manual 10. ARIC study website. 1991. http:// www.cscc.unc.edu/aric/pubuse/manual/Clinical_Chemistry_Determinations.1_10.pdf.

21. ARIC Coordinating Center. [Last accessed 15th June 2012] Atherosclerosis Risk in Communities Study Protocol: Cohort Component Procedures, Manual 2. ARIC study website. 1997. http:// www.cscc.unc.edu/aric/pubuse/manual/Cohort_Procedures.1_2.pdf.

22. UCLA Academic Technology Services. Analyzing Data with a Stratified Sampling Design: Stata 9. Texas: Stata Computing, College Station; 2006.

23. Astor BC, Sharrett AR, Coresh J, Chambless LE, Wasserman BA. Remodeling of carotid arteries detected with MR imaging: atherosclerosis risk in communities carotid MRI study. Radiology. 2010; 256(3):879-886. [PubMed: 20651061]

24. Wagenknecht L, Wasserman B, Chambless L, Coresh J, Folsom A, Mosley T, et al. Correlates of carotid plaque presence and composition as measured by MRI: the Atherosclerosis Risk in Communities Study. Circ Cardiovasc Imaging. 2009; 2(4):314-322. [PubMed: 19808612]

25. Saba L, Sanfilippo R, Pascalis L, Montisci R, Caddeo G, Mallarini G. Carotid artery wall thickness and ischemic symptoms: evaluation using multi-detector-row CT angiography. Eur Radiol. 2008; 18(9):1962-1971. [PubMed: 18404269]

26. Zureik M, Ducimetiere P, Touboul PJ, Courbon D, Bonithon-Kopp C, Berr C, et al. Common carotid intima-media thickness predicts occurrence of carotid atherosclerotic plaques: longitudinal results from the aging vascular study (EVA). Aterioscler Thromb Vasc Bio. 2000; 20(6):16221629.

27. Inoue K, Matsumoto M, Shono T, Toyokawa S, Moriki A. Increased intima-media thickness and atherosclerotic plaque in the carotid artery as risk factors for silent brain infarcts. J Stroke Cerebrovasc Dis. 2007; 16:14-20. [PubMed: 17689386]

28. Pasterkamp G, Schoneveld AH, van der Wal AC, Haudenschild CC, Clarijs RJ, Becker AE, et al. Relation of arterial geometry to luminal narrowing and histologic markers for plaque vulnerability: the remodeling paradox. J Am Coll Cardiol. 1998; 32:655-662. [PubMed: 9741507]

29. Naghavi M, Libby P, Falk E, Casscells SW, Litovsky S, Rumberger J, et al. From vulnerable plaque to vulnerable patient: a call for new definitions and risk assessment strategies: Part I. Circulation. 2003; 108:1664-1672. [PubMed: 14530185]

30. Kelley DE, Slasky BS, Janosky J. Skeletal muscle density: effects of obesity and non-insulindependent diabetes mellitus. Am J Clin Nutr. 1991; 54:509-515. [PubMed: 1877507]

31. Del PS, Bonadonna RC, Bonora E Gulli G, Solini A, Shank M, et al. Characterization of cellular defects of insulin action in type 2 (non- insulin-dependent) diabetes mellitus. J Clin Invest. 1993; 91:484-494. [PubMed: 8432857]

32. Morino K, Petersen KF, Dufour S Befroy D, Frattini J, Shatzkes N, et al. Reduced mitochondrial density and increased IRS-1 serine phosphorylation in muscle of insulin-resistant offspring of type 2 diabetic parents. J Clin Invest. 2005; 115:3587-3593. [PubMed: 16284649]

33. Kelley DE, He J, Menshikova EV, Ritov VB. Dysfunction of mitochondria in human skeletal muscle in type 2 diabetes. Diabetes. 2002; 51:2944-2950. [PubMed: 12351431]

34. Patti ME, Butte AJ, Crunkhorn S Cusi K, Berria R, Kashyap S, et al. Coordinated reduction of genes of oxidative metabolism in humans with insulin resistance and diabetes: Potential role of PGC1 and NRF1. Proc Natl Acad Sci USA. 2003; 100:8466-8471. [PubMed: 12832613]

35. Mootha VK, Lindgren CM, Eriksson KF, Subramanian A, Sihag S, Lehar J, et al. PGC-1alpharesponsive genes involved in oxidative phosphorylation are coordinately downregulated in human diabetes. Nat Genet. 2003; 34:267-273. [PubMed: 12808457]

36. Petersen KF, Dufour S, Befroy D, Garcia R, Shulman GI. Impaired mitochondrial activity in the insulin-resistant offspring of patients with type 2 diabetes. N Engl J Med. 2004; 350:664-671. [PubMed: 14960743] 
37. Bruce CR, Anderson MJ, Carey AL, Newman DG, Bonen A, Kriketos AD, et al. Muscle oxidative capacity is a better predictor of insulin sensitivity than lipid status. J Clin Endocrinol Metab. 2003; 88:5444-5451. [PubMed: 14602787]

38. Lowell BB, Shulman GI. Mitochondrial dysfunction and type 2 diabetes. Science. 2005; 307:384387. [PubMed: 15662004] 


\section{Highlights}

- Blood lactate is associated with atherosclerosis measured using carotid MRI

- The association is strong and independent of other cardiovascular risk factors

- Mitochondrial dysfunction mediates the role of insulin resistance on atherosclerosis 


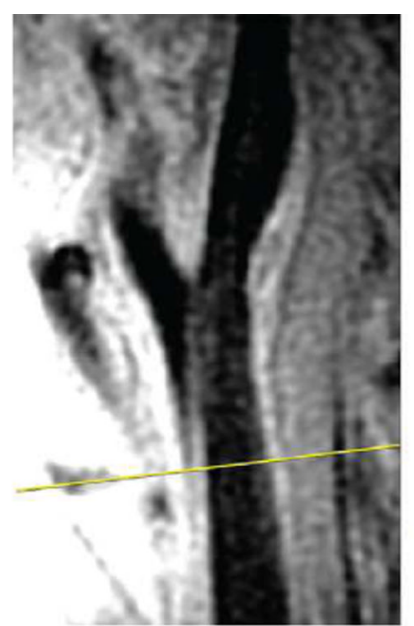

a.

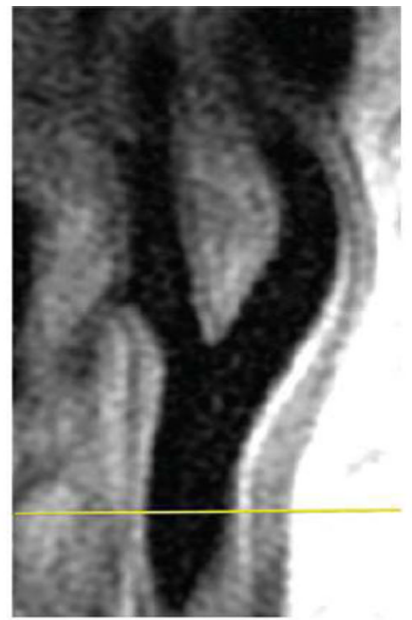

b.

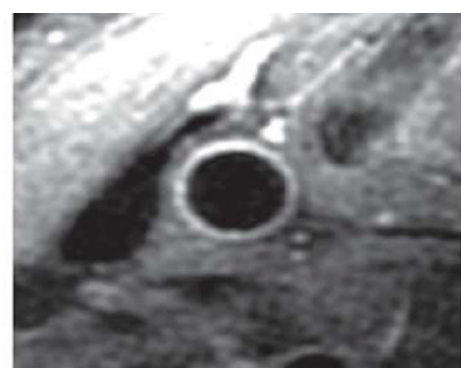

c.

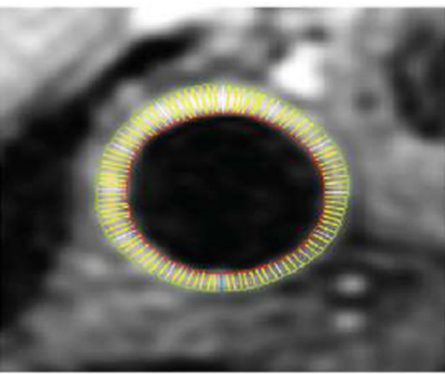

e.

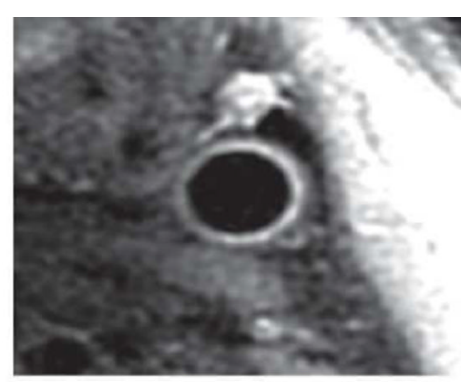

d.

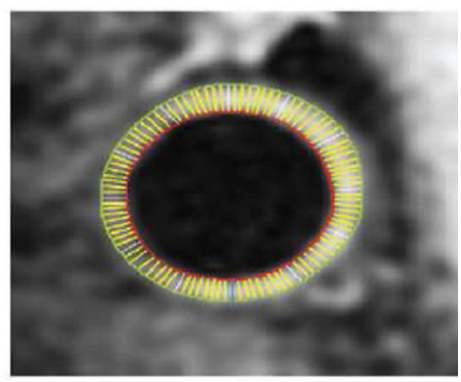
f.

Figure 1. MR angiogram as a scout image

Image is of the CCAs of a 71 year-old male ARIC Carotid MRI participant (Participant ID is J290624). Magnetic Resonance angiogram showing scout film: a, b: the red line indicates a reference land mark $15 \mathrm{~mm}$ below the carotid bifurcation where the MRI variable maximum wall thickness was measured. Further, c, d, e, f represents cross-sectional images of the carotid wall at the reference line depicted in $\mathrm{a}, \mathrm{b}$ where maximum wall thickness was measured. 


\begin{tabular}{|c|c|c|c|c|c|c|c|c|c|c|c|c|c|c|c|}
\hline $\bar{\Sigma}$ & & 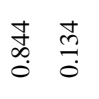 & $\begin{array}{l}\bar{B} \\
\dot{\square} \\
\dot{v}\end{array}$ & 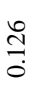 & $\begin{array}{ll}\stackrel{0}{\circ} \\
0 \\
0\end{array}$ & $\begin{array}{l}\overline{8} \\
\dot{\leftrightarrow}\end{array}$ & $\begin{array}{l}\overline{8} \\
\dot{\nabla}\end{array}$ & $\begin{array}{ll}\bar{\delta} & \overline{8} \\
\dot{0} & \\
\dot{v} & \dot{v}\end{array}$ & $\overbrace{0}^{n} \frac{n}{0}$ & 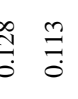 & $\begin{array}{l}\overline{8} \\
\dot{+} \\
\dot{v}\end{array}$ & $\begin{array}{l}0 \\
\exists \\
0 \\
\end{array}$ & $\stackrel{\infty}{\stackrel{\infty}{0}} \stackrel{\overline{0}}{0}$ & $\begin{array}{l}\bar{Q} \\
\dot{\sigma}\end{array}$ & $\begin{array}{ll}\bar{\delta} & \overline{8} \\
\dot{\theta} & \dot{v}\end{array}$ \\
\hline & 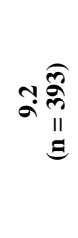 & 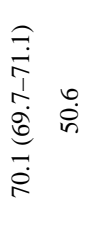 & $\underset{\dot{d}}{\stackrel{0}{\sim}}$ & & 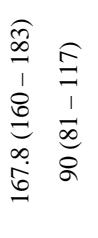 & 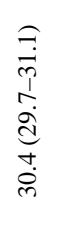 & ○े & 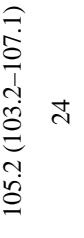 & 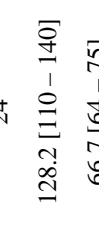 & 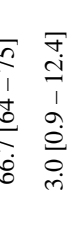 & $\stackrel{\rho}{m}$ & 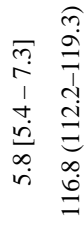 & 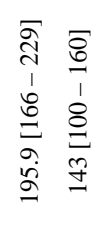 & $\begin{array}{l}\hat{\sigma} \\
\dot{\sigma} \\
\infty \\
\infty \\
\dot{g} \\
\hat{\sigma} \\
\hat{\sigma}\end{array}$ & 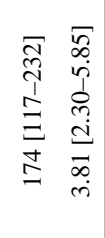 \\
\hline 急 & 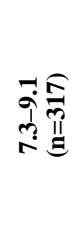 & 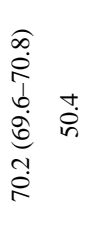 & $\stackrel{n}{2}$ & & 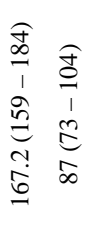 & 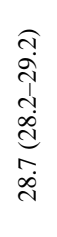 & m & 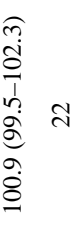 & 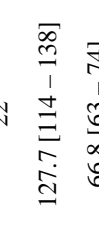 & 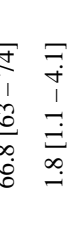 & $\stackrel{\sim}{\sim}$ & 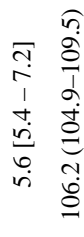 & 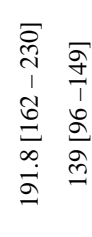 & 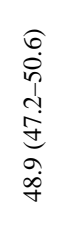 & 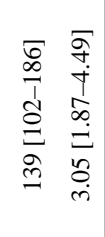 \\
\hline 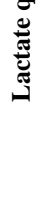 & 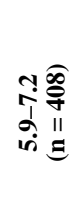 & 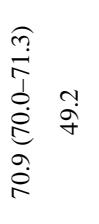 & $\vec{\infty}$ & $\stackrel{\rho}{\infty}$ & 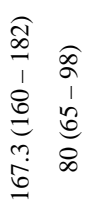 & 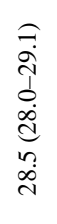 & $\begin{array}{l} \\
\infty \\
i \\
j \\
j\end{array}$ & 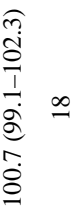 & 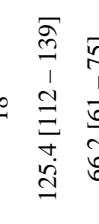 & 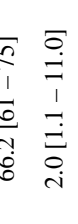 & 苢 & 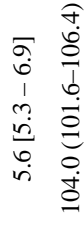 & 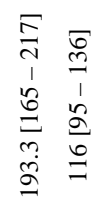 & 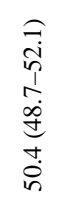 & 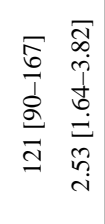 \\
\hline & 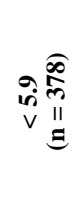 & 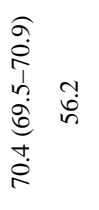 & $\stackrel{\circ}{\stackrel{j}{\longrightarrow}}$ & & 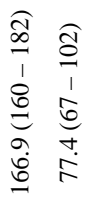 & 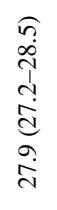 & ì. & 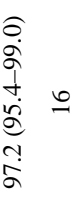 & 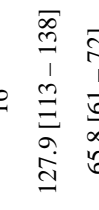 & 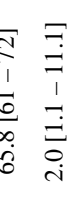 & $\stackrel{\leftrightarrow}{=}$ & 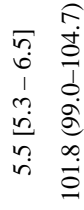 & 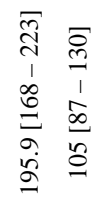 & 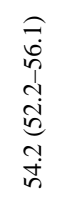 & 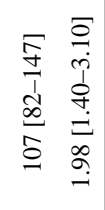 \\
\hline$=$ & & 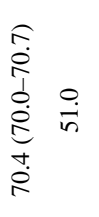 & & & 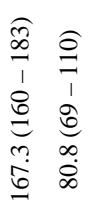 & 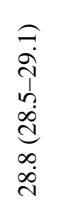 & & 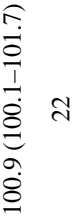 & 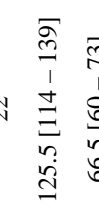 & 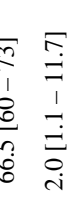 & ڤே. & 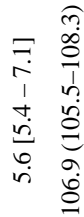 & 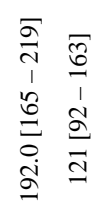 & 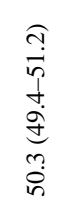 & 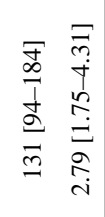 \\
\hline & & 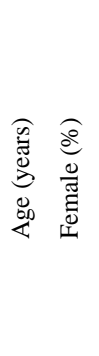 & 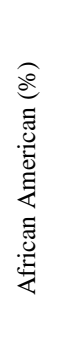 & 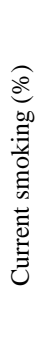 & 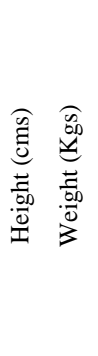 & 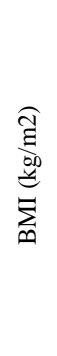 & 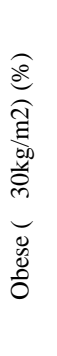 & 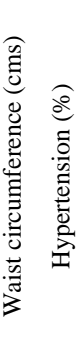 & 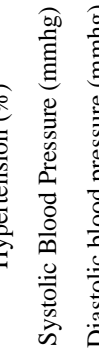 & 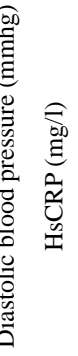 & 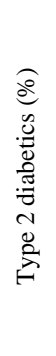 & 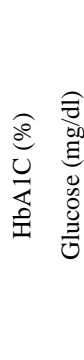 & 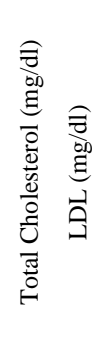 & 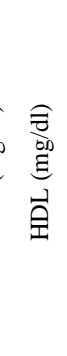 & 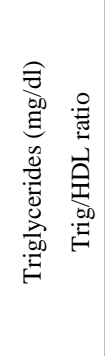 \\
\hline
\end{tabular}




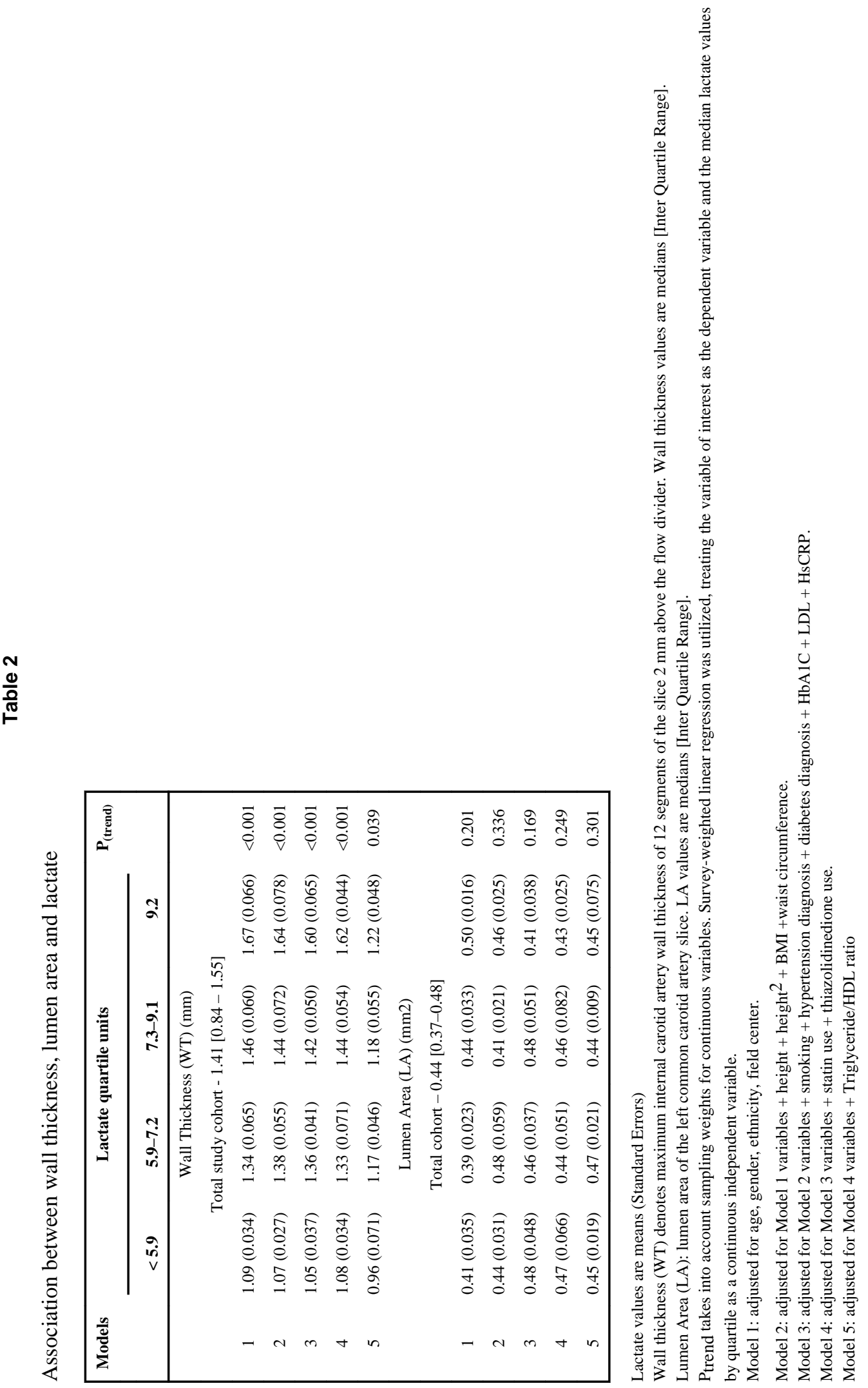

Atherosclerosis. Author manuscript; available in PMC 2014 May 01. 